\title{
New approach to prove the existence of classical solutions for a class of nonlinear parabolic equations
}

\author{
Svetlin G. Georgiev ${ }^{1}$ and Khaled ZenniR ${ }^{2}$ \\ ${ }^{1}$ Sorbonne University, Paris, France, \\ University of Sofia, Faculty of Mathematics and Informatics, \\ Department of Differential Equations. \\ ${ }^{2}$ Department Of Mathematics, \\ College Of Sciences and Arts, Al-Ras. \\ Qassim University, \\ Kingdom Of Saudi Arabia. \\ svetlingeorgiev1@gmail.com, sgg2000bg@yahoo.com, khaledzennir2@yahoo.com
}

\begin{abstract}
In this article, we consider a class of nonlinear parabolic equations. We use an integral representation combined with a sort of fixed point theorem to prove the existence of classical solutions for the initial value problem (1.1), (1.2). We also obtain a result on continuous dependence on the initial data. We propose a new approach for investigation for existence of classical solutions of some classes nonlinear parabolic equations.
\end{abstract}

\section{RESUMEN}

En este artículo, consideramos una clase de ecuaciones parabólicas nolineales. Usamos una representación integral combinada con una especie de teorema de punto fijo para probar la existencia de soluciones clásicas para el problema de valor inicial (1.1), (1.2). También obtenemos un resultado sobre la dependencia continua de la data inicial. Proponemos una estrategia nueva para la investigación de la existencia de soluciones clásicas de algunas clases de ecuaciones parabólicas nolineales.

Keywords and Phrases: parabolic equation, existence, differentiability with respect to the initial data

2010 AMS Mathematics Subject Classification: 35K55, 35K45. 


\section{Introduction}

Here, we consider the Cauchy problem

$$
\begin{aligned}
& u_{t}-u_{x x}=f\left(t, x, u, u_{x}\right) \text { in } \quad(0, \infty) \times \mathbb{R}, \\
& u(0, x)=\phi(x) \quad \text { in } \quad \mathbb{R}
\end{aligned}
$$

where $\phi \in \mathcal{C}^{2}(\mathbb{R}), f:[0, \infty) \times \mathbb{R} \times \mathbb{R} \times \mathbb{R} \longmapsto \mathbb{C}$ is a given continuous function, $u:[0, \infty) \times \mathbb{R} \longmapsto \mathbb{C}$ is the main unknown.

Our main results are as follows.

Theorem 1.1. Let $f \in \mathcal{C}([0, \infty) \times \mathbb{R} \times \mathbb{R} \times \mathbb{R}), \phi \in \mathcal{C}^{2}(\mathbb{R})$. Then there exists $\mathrm{m} \in(0,1)$ such that the problem (1.1), (1.2) has a solution $\mathrm{u} \in \mathcal{C}^{1}\left([0, \mathrm{~m}], \mathcal{C}^{2}([0,1])\right)$.

Theorem 1.2. Let $f \in \mathcal{C}([0, \infty) \times \mathbb{R} \times \mathbb{R} \times \mathbb{R}), \phi \in \mathcal{C}^{2}(\mathbb{R})$. Then there exists $\mathrm{m} \in(0,1)$ such that the problem (1.1), (1.2) has a solution $u \in \mathcal{C}^{1}\left([0, \mathrm{~m}], \mathcal{C}^{2}(\mathbb{R})\right)$.

For $\mathrm{O}_{1}, \mathrm{O}_{2} \subset \mathbb{R}$ with $\mathcal{C}^{1}\left(\mathrm{O}_{1}, \mathcal{C}^{2}\left(\mathrm{O}_{2}\right)\right)$ we denote the space of all continuous functions $\mathrm{u}$ on $\mathrm{O}_{1} \times \mathrm{O}_{2}$ such that $u_{t}, u_{x}$ and $u_{x x}$ exist and are continuous on $\mathrm{O}_{1} \times \mathrm{O}_{2}$.

Example 1.3. Let $\mathrm{p}>1$ and $\mathrm{a} \in \mathbb{C}$ be chosen so that $\mathrm{a}^{\mathrm{p}-1}=-\frac{1}{\mathrm{p}-1}$. Consider the Cauchy problem

$$
\begin{aligned}
& \mathfrak{u}_{\mathrm{t}}-\mathrm{u}_{\mathrm{xx}}=\mathrm{u}^{\mathrm{p}} \text { in }(0, \infty) \times \mathbb{R} \\
& \mathfrak{u}(0, x)=\mathrm{a} \text { in } \mathbb{R} .
\end{aligned}
$$

Then $\mathfrak{u}(\mathrm{t}, \mathrm{x})=\mathrm{a}(\mathrm{t}+1)^{-\frac{1}{\mathrm{p}-1}}$ is its solution. Actually,

$$
u_{t}(t, x)=-\frac{a}{p-1}(t+1)^{-\frac{p}{p-1}}
$$

and

$$
u_{x x}(t, x)=0
$$

and

$$
(u(t, x))^{p}=-\frac{a}{p-1}(t+1)^{-\frac{p}{p-1}}
$$

Therefore

$$
u_{t}(t, x)-u_{x x}(t, x)=(u(t, x))^{p} \quad \text { in } \quad(0, \infty) \times \mathbb{R}
$$

and

$$
u(0, x)=a \quad \text { in } \quad \mathbb{R} .
$$


To prove our main result we propose new integral representation of the solutions of the initial value problem (1.1), (1.2). Many works have been devoted to the investigation of initial value problems for parabolic equations and systems (see, for example, [13-16] and the references therein). We note that in the references the IVP (1.1), (1.2) is connected with the dimension n, Fujita exponent, Sobolev critical exponents, bounded and unbounded domain. In this article we propose new idea which tell us that the local existence of classical solutions of the IVP is connected with the integral representation of the solutions, it is not connected with the dimension $n$ and if the domain is bounded or not.

As an application of our new integral representation we deduce some results connected with the continuous dependence on the initial data and parameters of the problem (1.1), (1.2).

Theorem 1.4. Let $\mathrm{f} \in \mathcal{C}([0, \infty) \times \mathbb{R} \times \mathbb{R} \times \mathbb{R}), \frac{\partial \mathrm{f}}{\partial \mathfrak{u}}$, $\frac{\partial \mathrm{f}}{\partial \mathfrak{u}_{x}}$ exist and are continuous in $[0, \infty) \times \mathbb{R} \times \mathbb{R} \times \mathbb{R}$, $\phi \in \mathcal{C}^{2}(\mathbb{R})$. Let also, $\mathrm{u}(\mathrm{t}, \mathrm{x}, \phi) \in \mathcal{C}^{1}\left([0, \mathrm{~m}], \mathcal{C}^{2}([\mathrm{c}, \mathrm{d}])\right)$ be a solution to the problem (1.1), (1.2) for some $\mathrm{m} \in(0,1)$ and for some $[\mathrm{c}, \mathrm{d}] \subset \mathbb{R}$. Then $\mathrm{u}(\mathrm{t}, \mathrm{x}, \phi)$ is differentiable with respect to $\phi$ and $v(t, x)=\frac{\partial u}{\partial \phi}(t, x, \phi)$ satisfies the following initial value problem

$$
\begin{aligned}
v_{t}-v_{x x}= & \frac{\partial f}{\partial u}\left(t, x, u(t, x, \phi), u_{x}(t, x, \phi)\right) v \\
& +\frac{\partial f}{\partial u_{x}}\left(t, x, u(t, x, \phi), u_{x}(t, x, \phi)\right) v_{x} \quad \text { in }[0, m] \times[c, d], \\
v & (0, x)=1 \quad \text { in }[c, d] .
\end{aligned}
$$

\section{Auxiliary results}

We will start with the following useful lemma.

Lemma 2.1. Let $\mathrm{f} \in \mathcal{C}([\mathrm{a}, \mathrm{b}] \times[\mathrm{c}, \mathrm{d}] \times \mathbb{R} \times \mathbb{R}), \mathrm{g} \in \mathcal{C}^{2}([\mathrm{c}, \mathrm{d}])$. Then the function $\mathrm{u} \in \mathcal{C}^{1}\left([\mathrm{a}, \mathrm{b}], \mathcal{C}^{2}([\mathrm{c}, \mathrm{d}])\right)$ is a solution to the problem

$$
\begin{array}{r}
u_{t}-u_{x x}=f\left(t, x, u, u_{x}\right) \quad \text { in } \quad(a, b] \times[c, d], \\
u(a, x)=g(x) \quad \text { in } \quad[c, d],
\end{array}
$$

if and only if it is a solution to the integral equation

$$
\begin{aligned}
& \int_{c}^{x} \int_{c}^{y}(u(t, z)-g(z)) d z d y-\int_{a}^{t}\left(u(\tau, x)-u(\tau, c)-(x-c) u_{x}(\tau, c)\right) d \tau \\
& =\int_{a}^{t} \int_{c}^{x} \int_{c}^{y} f\left(\tau, z, u(\tau, z), u_{x}(\tau, z)\right) d z d y d \tau, \quad x \in[c, d], \quad t \in[a, b] .
\end{aligned}
$$

Proof. (1) Let $u \in \mathcal{C}^{1}\left([a, b], \mathcal{C}^{2}([c, d])\right)$ is a solution to the problem (2.1), (2.2).

We integrate the equation (2.1) with respect to $x$ and we get

$$
\begin{aligned}
& \int_{c}^{x} u_{t}(t, z) d z-\int_{c}^{x} u_{x x}(t, z) d z \\
& =\int_{c}^{x} f\left(t, z, u(t, z), u_{x}(t, z)\right) d z, \quad x \in[c, d], \quad t \in[a, b],
\end{aligned}
$$


or

$$
\begin{aligned}
& \int_{c}^{x} u_{t}(t, z) d z-u_{x}(t, x)+u_{x}(t, c) \\
& =\int_{c}^{x} f\left(t, z, u(t, z), u_{x}(t, z)\right) d z, \quad x \in[c, d], \quad t \in[a, b] .
\end{aligned}
$$

Now we integrate the last equation with respect to $x$ and we find

$$
\begin{aligned}
& \int_{c}^{x} \int_{c}^{y} u_{t}(t, z) d z d y-\int_{c}^{x}\left(u_{x}(t, z)-u_{x}(t, c)\right) d z \\
& =\int_{c}^{x} \int_{c}^{y} f\left(t, z, u(t, z), u_{x}(t, z)\right) d z d y, \quad x \in[c, d], \quad t \in[a, b],
\end{aligned}
$$

or

$$
\begin{aligned}
& \int_{c}^{x} \int_{c}^{y} u_{t}(t, z) d z d y-u(t, x)+u(t, c)+(x-c) u_{x}(t, c) \\
& =\int_{c}^{x} \int_{c}^{y} f\left(t, z, u(t, z), u_{x}(t, z)\right) d z d y, \quad x \in[c, d], \quad t \in[a, b] .
\end{aligned}
$$

We integrate the last equality with respect to $t$ and we obtain

$$
\begin{aligned}
& \int_{a}^{t} \int_{c}^{x} \int_{c}^{y} u_{t}(s, z) d z d y d s-\int_{a}^{t}\left(u(s, x)-u(s, c)-(x-c) u_{x}(s, c)\right) d s \\
& =\int_{a}^{t} \int_{c}^{x} \int_{c}^{y} f\left(s, z, u(s, z), u_{x}(s, z)\right) d z d y d s, \quad x \in[c, d], \quad t \in[a, b],
\end{aligned}
$$

or

$$
\begin{aligned}
& \int_{c}^{x} \int_{c}^{y}(u(t, z)-g(z)) d z d y-\int_{a}^{t}\left(u(s, x)-u(s, c)-(x-c) u_{x}(s, c)\right) d s \\
& =\int_{a}^{t} \int_{c}^{x} \int_{c}^{y} f\left(s, z, u(s, z), u_{x}(s, z)\right) d z d y d s, \quad x \in[c, d], \quad t \in[a, b],
\end{aligned}
$$

i.e., $u$ satisfies the equation (2.3).

(2) Let $u \in \mathcal{C}^{1}\left([a, b], \mathcal{C}^{2}([c, d])\right)$ be a solution to the integral equation (2.3).

We differentiate the equation (2.3) with respect to $x$ and we get

$$
\begin{aligned}
& \int_{c}^{x}(u(t, z)-g(z)) d z-\int_{a}^{t}\left(u_{x}(s, x)-u_{x}(s, c)\right) d s \\
& =\int_{a}^{t} \int_{c}^{x} f\left(s, z, u(s, z), u_{x}(s, z)\right) d z d s, \quad x \in[c, d], \quad t \in[a, b] .
\end{aligned}
$$

Again we differentiate with respect to $x$ and we find

$$
\begin{aligned}
& u(t, x)-g(x)-\int_{a}^{t} u_{x x}(s, x) d s \\
& =\int_{a}^{t} f\left(s, x, u(s, x), u_{x}(s, x)\right) d s, \quad x \in[c, d], \quad t \in[a, b] .
\end{aligned}
$$

Now we put $t=a$ in the last equation and we find

$$
u(a, x)=g(x), \quad x \in[c, d]
$$


i.e., the function $u$ satisfies (2.2).

Now we differentiate the equation (2.4) with respect to $t$ and we find

$$
u_{t}(t, x)-u_{x x}(t, x)=f\left(t, x, u(t, x), u_{x}(t, x)\right), \quad x \in[c, d], t \in[a, b] .
$$

The proof of the existence results are based on the following theorem.

Theorem 2.2 ([14]). Let $\mathrm{X}$ be a nonempty closed convex subset of a Banach space $\mathrm{Y}$. Suppose that $\mathrm{T}$ and $\mathrm{S}$ map $\mathrm{X}$ into $\mathrm{Y}$ such that

(1) $\mathrm{S}$ is continuous and $\mathrm{S}(\mathrm{X})$ contained in a compact subset of $\mathrm{Y}$.

(2) $\mathrm{T}: \mathrm{X} \longmapsto \mathrm{Y}$ is expansive and onto.

Then there exists a point $\chi^{*} \in X$ such that

$$
\mathrm{Sx} \mathrm{x}^{*}+\mathrm{Tx}^{*}=\mathrm{x}^{*} .
$$

Definition 2.3. Let $(X, d)$ be a metric space and $M$ be a subset of $X$. The mapping $T: M \longmapsto X$ is said to be expansive if there exists a constant $\mathrm{h}>1$ such that

$$
d(T x, T y) \geq h d(x, y)
$$

for any $\mathrm{x}, \mathrm{y} \in \mathrm{M}$.

\section{Proof of Theorem 1.1}

Let $B>\|\phi\|_{\mathcal{C}^{2}([0,1])}$ be arbitrarily chosen. Since $\phi \in \mathcal{C}([0,1]), f \in \mathcal{C}([0,1] \times[0,1] \times[-B, B] \times[-B, B])$ we have that there exists a constant $M_{11}>0$ such that

$$
\begin{aligned}
|\phi(x)| & \leq M_{11} \quad \text { in } \quad[0,1], \\
|f(t, x, y, z)| & \leq M_{11} \quad \text { in }[0,1] \times[0,1] \times[-B, B] \times[-B, B] .
\end{aligned}
$$

We take $l, m \in(0,1)$ so that

$$
\begin{aligned}
& l B+l\left(B+M_{11}\right)+3 l B m+l M_{11} m \leq B \\
& l\left(5 B+2 M_{11}\right) \leq B .
\end{aligned}
$$

Let $E_{11}=\mathcal{C}^{1}\left([0, m], \mathcal{C}^{2}([0,1])\right)$ be endowed with the norm

$$
\begin{aligned}
& \|u\|=\max \left\{\max _{(t, x) \in[0, m] \times[0,1]}|\mathfrak{u}(t, x)|, \quad \max _{(t, x) \in[0, m] \times[0,1]}\left|u_{t}(t, x)\right|,\right. \\
& \left.\max _{(t, x) \in[0, m] \times[0,1]}\left|u_{x}(t, x)\right|, \quad \max _{(t, x) \in[0, m] \times[0,1]}\left|u_{x x}(t, x)\right|\right\} .
\end{aligned}
$$


By $\tilde{\mathrm{K}}_{11}$ we denote the set of all equi-continuous families in $E_{11}$, i.e., for every $\epsilon>0$ there exists $\delta=\delta(\epsilon)>0$ such that

$$
\begin{aligned}
\left|u\left(t_{1}, x_{1}\right)-u\left(t_{2}, x_{2}\right)\right|<\epsilon, \quad & \left|u_{t}\left(t_{1}, x_{1}\right)-u_{t}\left(t_{2}, x_{2}\right)\right|<\epsilon, \\
\left|u_{x}\left(t_{1}, x_{1}\right)-u_{x}\left(t_{2}, x_{2}\right)\right|<\epsilon, \quad & \left|u_{x x}\left(t_{1}, x_{1}\right)-u_{x x}\left(t_{2}, x_{2}\right)\right|<\epsilon
\end{aligned}
$$

whenever $\left|t_{1}-t_{2}\right|<\delta,\left|x_{1}-x_{2}\right|<\delta$. Let also,

$$
\mathrm{K}_{11}^{\prime}=\overline{\tilde{\mathrm{K}}_{11}}, \quad \mathrm{~K}_{11}=\left\{\mathrm{u} \in \mathrm{K}_{11}^{\prime}:\|\mathrm{u}\| \leq \mathrm{B}\right\}
$$

and

$$
\mathrm{L}_{11}=\left\{u \in \mathrm{K}_{11}^{\prime}:\|u\| \leq(1+\mathrm{l}) \mathrm{B}\right\}
$$

We note that $K_{11}$ is a closed convex subset of $L_{11}$.

For $u \in \mathrm{L}_{11}$ we define the operators

$$
\begin{aligned}
T_{11}(u)(t, x)= & (1+l) u(t, x) \\
S_{11}(u)(t, x)= & -l u(t, x)+l \int_{0}^{x} \int_{0}^{y}(u(t, z)-\phi(z)) d z d y \\
& -l \int_{0}^{t}\left(u(\tau, x)-u(\tau, 0)-x u_{x}(\tau, 0)\right) d \tau \\
& -l \int_{0}^{t} \int_{0}^{x} \int_{0}^{y} f\left(\tau, z, u(\tau, z), u_{x}(\tau, z)\right) d z d y d \tau .
\end{aligned}
$$

We will prove that the problem

$$
\begin{gathered}
u_{t}-u_{x x}=f\left(t, x, u_{x}\right) \text { in }[0, m] \times[0,1] \\
u(0, x)=\phi(x) \text { in }[0,1]
\end{gathered}
$$

has a solution $u \in \mathcal{C}^{1}\left([0, m], \mathcal{C}^{2}([0,1])\right)$.

a) $S_{11}: K_{11} \longmapsto K_{11}$. Let $u \in K_{11}$. Then $S_{11}(u) \in \mathcal{C}^{1}\left([0, m], \mathcal{C}^{2}([0,1])\right)$ and for $(t, x) \in[0, m] \times$ 
$[0,1]$, using the first inequality of (3.1), we get

$$
\begin{aligned}
\left|S_{11}(u)(t, x)\right|= & \mid-l u(t, x)+l \int_{0}^{x} \int_{0}^{y}(u(t, z)-\phi(z)) d z d y \\
& -l \int_{0}^{t}\left(u(\tau, x)-u(\tau, 0)-x u_{x}(\tau, 0)\right) d \tau \\
& -l \int_{0}^{t} \int_{0}^{x} \int_{0}^{y} f\left(\tau, z, u(\tau, z), u_{x}(\tau, z)\right) d z d y d \tau \mid \\
\leq & l|u(t, x)|+l \int_{0}^{x} \int_{0}^{y}(|u(t, z)|+|\phi(z)|) d z d y \\
& +l \int_{0}^{t}\left(|u(\tau, x)|+|u(\tau, 0)|+x\left|u_{x}(\tau, 0)\right|\right) d \tau \\
\leq \quad & \quad \text { B. } \int_{0}^{t} \int_{0}^{x} \int_{0}^{y}\left|f\left(\tau, z, u(\tau, z), u_{x}(\tau, z)\right)\right| d z d y d \tau \\
\leq & +l\left(B+M_{11}\right)+3 l B m+l M_{11} m
\end{aligned}
$$

Note that

$$
\begin{aligned}
S_{11}(u)_{t}(t, x)= & -l u_{t}(t, x)+l \int_{0}^{x} \int_{0}^{y} u_{t}(t, z) d z d y \\
& -l\left(u(t, x)-u(t, 0)-x u_{x}(t, 0)\right) \\
& -l \int_{0}^{x} \int_{0}^{y} f\left(t, z, u(t, z), u_{x}(t, z)\right) d z d y \\
& (t, x) \in[0, m] \times[0,1] .
\end{aligned}
$$


Then, using the second inequality of (3.1), we obtain

$$
\begin{aligned}
\left|S_{11}(u)_{t}(t, x)\right|= & \mid-l u_{t}(t, x)+l \int_{0}^{x} \int_{0}^{y} u_{t}(t, z) d z d y \\
& -l\left(u(t, x)-u(t, 0)-x u_{x}(t, 0)\right) \\
& -l \int_{0}^{x} \int_{0}^{y} f\left(t, z, u(t, z), u_{x}(t, z)\right) d z d y \mid \\
\leq & l\left|u_{t}(t, x)\right|+l \int_{0}^{x} \int_{0}^{y}\left|u_{t}(t, z)\right| d z d y \\
& +l\left(|u(t, x)|+|u(t, 0)|+x\left|u_{x}(t, 0)\right|\right) \\
& +l \int_{0}^{x} \int_{0}^{y}\left|f\left(t, z, u(t, z), u_{x}(t, z)\right)\right| d z d y \\
\leq & l B+l B+3 l B+l M_{11} \\
= & l\left(5 B+M_{11}\right) \\
\leq & B, \quad(t, x) \in[0, m] \times[0,1] .
\end{aligned}
$$

Also,

$$
\begin{aligned}
S_{11}(u)_{x}(t, x)= & -l u_{x}(t, x)+l \int_{0}^{x}(u(t, z)-\phi(z)) d z \\
& -l \int_{0}^{t}\left(u_{x}(\tau, x)-u_{x}(\tau, 0)\right) d \tau \\
& -l \int_{0}^{t} \int_{0}^{x} f\left(\tau, z, u(\tau, z), u_{x}(\tau, z)\right) d z d \tau, \\
& (t, x) \in[0, m] \times[0,1] .
\end{aligned}
$$


Hence, using the first inequality of (3.1),

$$
\begin{aligned}
\left|S_{11}(u)_{x}(t, x)\right|= & \mid-l u_{x}(t, x)+l \int_{0}^{x}(u(t, z)-\phi(z)) d z \\
& -l \int_{0}^{t}\left(u_{x}(\tau, x)-u_{x}(\tau, 0)\right) d \tau \\
& -l \int_{0}^{t} \int_{0}^{x} f\left(\tau, z, u(\tau, z), u_{x}(\tau, z)\right) d z d \tau \mid \\
\leq & l\left|u_{x}(t, x)\right|+l \int_{0}^{x}(|u(t, z)|+|\phi(z)|) d z \\
& +l \int_{0}^{t}\left(\left|u_{x}(\tau, x)\right|+\left|u_{x}(\tau, 0)\right|\right) d \tau \\
& +l \int_{0}^{t} \int_{0}^{x}\left|f\left(\tau, z, u(\tau, z), u_{x}(\tau, z)\right)\right| d z d \tau \\
\leq & l B+l\left(B+M_{11}\right)+2 l B m+l M_{11} m \\
\leq & B, \quad(t, x) \in[0, m] \times[0,1] .
\end{aligned}
$$

For $(t, x) \in[0, m] \times[0,1]$ we have

$$
\begin{aligned}
S_{11}(u)_{x x}(t, x)= & -l u_{x x}(t, x)+l(u(t, x)-\phi(x)) \\
& -l \int_{0}^{t} u_{x x}(\tau, x) d \tau \\
& -l \int_{0}^{t} f\left(\tau, x, u(\tau, x), u_{x}(\tau, x)\right) d \tau
\end{aligned}
$$


from where, using the first inequality of (3.1),

$$
\begin{aligned}
\left|S_{11}(u)_{x x}(t, x)\right|= & \mid-l u_{x x}(t, x)+l(u(t, x)-\phi(x)) \\
& -l \int_{0}^{t} u_{x x}(\tau, x) d \tau \\
& -l \int_{0}^{t} f\left(\tau, x, u(\tau, x), u_{x}(\tau, x)\right) d \tau \mid \\
\leq & l\left|u_{x x}(t, x)\right|+l(|u(t, x)|+|\phi(x)|) \\
& +l \int_{0}^{t}\left|u_{x x}(\tau, x)\right| d \tau \\
& +l \int_{0}^{t}\left|f\left(\tau, x, u(\tau, x), u_{x}(\tau, x)\right)\right| d \tau \\
\leq & l B+l\left(B+M_{11}\right)+l B m+l M_{11} m \\
\leq & B .
\end{aligned}
$$

We note that $\left\{S_{11}(u): u \in K_{11}\right\}$ is an equi-continuous family in $E_{11}$. Consequently $S_{11}$ : $\mathrm{K}_{11} \longmapsto \mathrm{K}_{11}$. Also, $\mathrm{S}_{11}\left(\mathrm{~K}_{11}\right) \subset \mathrm{K}_{11} \subset \mathrm{L}_{11}$, i.e., $\mathrm{S}_{11}\left(\mathrm{~K}_{11}\right)$ resides in a compact subset of $\mathrm{L}_{11}$.

b) $S_{11}: K_{11} \longmapsto K_{11}$ is a continuous operator. We note that if $\left\{u_{n}\right\}_{n=1}^{\infty}$ be a sequence of elements of $K_{11}$ such that $u_{n} \longrightarrow u$ in $K_{11}$ as $n \longrightarrow \infty$, then $S_{11}\left(u_{n}\right) \longrightarrow S_{11}(u)$ in $K_{11}$ as $\mathrm{n} \longrightarrow \infty$. Therefore $\mathrm{S}_{11}: \mathrm{K}_{11} \longmapsto \mathrm{K}_{11}$ is a continuous operator.

c) $T_{11}: K_{11} \longmapsto L_{11}$ is an expansive operator and onto. For $u, v \in K_{11}$ we have that

$$
\left\|\mathrm{T}_{11}(\mathrm{u})-\mathrm{T}_{11}(v)\right\|=(1+\mathrm{l})\|\mathrm{u}-v\|
$$

i.e., $\mathrm{T}_{11}: \mathrm{K}_{11} \longmapsto \mathrm{L}_{11}$ is an expansive operator with constant $1+l$.

Let $v \in \mathrm{L}_{11}$. Then $\frac{v}{1+\mathrm{l}} \in \mathrm{K}_{11}$ and

$$
\mathrm{T}_{11}\left(\frac{v}{1+l}\right)=v
$$

i.e., $\mathrm{T}_{11}: \mathrm{K}_{11} \longmapsto \mathrm{L}_{11}$ is onto.

From a), b), c) and from Theorem 2.2. it follows that there is $u_{11} \in K_{11}$ such that

$$
T_{11} u_{11}+S_{11} u_{11}=u_{11}
$$


or

$$
\begin{aligned}
& (1+l) u_{11}(t, x)-l u_{11}(t, x)+l \int_{0}^{x} \int_{0}^{y}\left(u_{11}(t, z)-\phi(z)\right) d z d y \\
& -l \int_{0}^{t}\left(u_{11}(\tau, x)-u_{11}(\tau, 0)-x u_{11 x}(\tau, 0)\right) d \tau \\
& \quad-l \int_{0}^{t} \int_{0}^{x} \int_{0}^{y} f\left(\tau, z, u_{11}(\tau, z), u_{11 x}(\tau, z)\right) d z d y d \tau \\
& =u_{11}(t, x)
\end{aligned}
$$

or

$$
\begin{aligned}
& \int_{0}^{x} \int_{0}^{y}\left(u_{11}(t, z)-\phi(z)\right) d z d y-\int_{0}^{t}\left(u_{11}(\tau, x)-u_{11}(\tau, 0)-x u_{11 x}(\tau, 0)\right) d \tau \\
& \quad-\int_{0}^{t} \int_{0}^{x} \int_{0}^{y} f\left(\tau, z, u_{11}(\tau, z), u_{11 x}(\tau, z)\right) d z d y d \tau \\
& =0, \quad(t, x) \in[0, m] \times[0,1]
\end{aligned}
$$

whereupon, using Lemma 2.1, we conclude that $u_{11} \in \mathcal{C}^{1}\left([0,1], \mathcal{C}^{2}([0,1])\right)$ is a solution to the problem (3.2), (3.3).

\section{Proof of Theorem 1.2}

Now we consider the problem

$$
\begin{gathered}
u_{t}-u_{x x}=f\left(t, x, u(t, x), u_{x}(t, x)\right) \quad \text { in }(0, m] \times[1,2], \\
u(0, x)=\phi(x) \quad \text { in }[1,2] .
\end{gathered}
$$

Let $E_{12}=\mathcal{C}^{1}\left([0, m], \mathcal{C}^{2}([1,2])\right)$ be endowed with the norm

$$
\begin{aligned}
&\|u\|= \max \left\{\max _{(t, x) \in[0, m] \times[1,2]}|u(t, x)|,\right. \\
& \max _{(t, x) \in[0, m] \times[1,2]}\left|u_{x}(t, x)\right|, \quad \max _{(t, x) \in[0, m] \times[1,2]}\left|u_{t}(t, x)\right|, \\
&\left.\max _{(t, x) \in[0, m] \times[1,2]}\left|u_{x x}(t, x)\right|\right\} .
\end{aligned}
$$

By $\tilde{K}_{12}$ we denote the set of all equi-continuous families in $E_{12}$.

Let $\mathrm{K}_{12}^{\prime}=\overline{\tilde{\mathrm{K}}_{12}}$,

$$
\mathrm{K}_{12}=\left\{\mathrm{u} \in \mathrm{K}_{12}^{\prime}:\|\mathrm{u}\| \leq \mathrm{B}\right\}
$$


Since $\phi \in \mathcal{C}([1,2]), f \in \mathcal{C}([0, m] \times[1,2] \times[-B, B] \times[-B, B])$ we have that there exists a constant $M_{12}>0$ such that

$$
\begin{aligned}
|\phi(x)| & \leq M_{12} \quad \text { in } \quad[1,2] \\
|f(t, x, y, z)| & \leq M_{12} \quad \text { in } \quad[0, m] \times[1,2] \times[-B, B] \times[-B, B] .
\end{aligned}
$$

Let $l_{1}>0$ be chosen so that

$$
\begin{aligned}
& l_{1}\left(5 B+2 M_{12}\right) \leq B \\
& l_{1} B+l_{1}\left(B+M_{12}\right)+3 l_{1} B m+l_{1} M_{12} m \leq B
\end{aligned}
$$

Let also,

$$
\mathrm{L}_{12}=\left\{u \in K_{12}^{\prime}:\|u\| \leq\left(1+l_{1}\right) B\right\}
$$

We note that $K_{12}$ is a closed convex subset of $L_{12}$.

For $u \in \mathrm{L}_{12}$ we define the operators

$$
\begin{aligned}
T_{12}(u)(t, x)= & \left(1+l_{1}\right) u(t, x), \\
S_{12}(u)(t, x)= & -l_{1} u(t, x)+l_{1} \int_{1}^{x} \int_{1}^{y}(u(t, z)-\phi(z)) d z d y \\
& -l_{1} \int_{0}^{t}\left(u(\tau, x)-u_{11}(\tau, 1)-(x-1) u_{11 x}(\tau, 1)\right) d \tau \\
& -l_{1} \int_{0}^{t} \int_{1}^{x} \int_{1}^{y} f\left(\tau, z, u(\tau, z), u_{x}(\tau, z)\right) d z d y d \tau .
\end{aligned}
$$

As in the previous section one can prove that there is $u_{12} \in \mathcal{C}^{1}\left([0,1], \mathcal{C}^{2}([1,2])\right)$ which is a solution to the problem (4.1), (4.2). This solution $\mathfrak{u}_{12}$ satisfies the integral equation

$$
\begin{aligned}
& \int_{1}^{x} \int_{1}^{y}\left(u_{12}(t, z)-\phi(z)\right) d z d y \\
& \quad-\int_{0}^{t}\left(u_{12}(\tau, x)-u_{11}(\tau, 1)-(x-1) u_{11 x}(\tau, 1)\right) d \tau \\
& \quad-\int_{0}^{t} \int_{1}^{x} \int_{1}^{y} f\left(\tau, z, u_{12}(\tau, z), u_{12 x}(\tau, z)\right) d z d y d \tau \\
& =0, \quad(t, x) \in[0, m] \times[1,2] .
\end{aligned}
$$

Now we put $x=1$ in 4.37 and we find

$$
\int_{0}^{t}\left(u_{12}(\tau, 1)-u_{11}(\tau, 1)\right) d \tau=0
$$


which we differentiate with respect to $t$ and we get

$$
u_{12}(t, 1)=u_{11}(t, 1) \quad \text { in } \quad[0, m] .
$$

Now we differentiate [4.3) with respect to $x$ and we find

$$
\begin{aligned}
& \int_{1}^{x}\left(u_{12}(t, z)-\phi(z)\right) d z-\int_{0}^{t}\left(u_{12 x}(\tau, x)-u_{11 x}(\tau, 1)\right) d \tau \\
& \quad-\int_{0}^{t} \int_{1}^{x} f\left(\tau, z, u_{12}(\tau, z), u_{12 x}(\tau, z)\right) d z d \tau=0, \quad(t, x) \in[0, m] \times[1,2] .
\end{aligned}
$$

In the last equation we put $x=1$ and we become

$$
\int_{0}^{t}\left(u_{12 x}(\tau, x)-u_{11 x}(\tau, 1)\right) d \tau=0, \quad(t, x) \in[0, m] \times[1,2]
$$

which we differentiate with respect to $t$ and we find

$$
u_{12 x}(t, 1)=u_{11 x}(t, 1) \quad \text { in } \quad[0, m] .
$$

Now we differentiate (4.4) with respect to $t$ and we get

$$
u_{12 t}(t, 1)=u_{11 t}(t, 1) \quad \text { in } \quad[0, m] .
$$

Hence, (4.4), 4.5) and

$$
f\left(t, 1, u_{11}(t, 1), u_{11 x}(t, 1)\right)=f\left(t, 1, u_{12}(t, 1), u_{12 x}(t, 1)\right),
$$

we find

$$
\begin{aligned}
u_{12 x x}(t, 1) & =u_{12 t}(t, 1)-f\left(t, 1, u_{12}(t, 1), u_{12 x}(t, 1)\right) \\
& =u_{11 t}(t, 1)-f\left(t, 1, u_{11}(t, 1), u_{11 x}(t, 1)\right) \\
& =u_{11 x x}(t, 1) \quad \text { in }[0, m] .
\end{aligned}
$$

Consequently the function

$$
u(t, x)=\left\{\begin{array}{lll}
u_{11}(t, x) & \text { in } & {[0, m] \times[0,1]} \\
u_{12}(t, x) & \text { in } & {[0, m] \times[1,2]}
\end{array}\right.
$$

is a $\mathcal{C}^{1}\left([0, \mathrm{~m}], \mathcal{C}^{2}([0,2])\right)$-solution to the problem

$$
\begin{aligned}
u_{t}-u_{x x} & =f\left(t, x, u(t, x), u_{x}(t, x)\right) \quad \text { in } \quad(0, m] \times[0,2], \\
u(0, x) & =\phi(x) \quad \text { in } \quad[0,2] .
\end{aligned}
$$


Then we consider the problem

$$
\begin{aligned}
& u_{t}-u_{x x}=f\left(t, x, u(t, x), u_{x}(t, x)\right) \quad \text { in } \quad(0, m] \times[2,3] \\
& u(0, x)=\phi(x) \quad \text { in }[2,3] .
\end{aligned}
$$

As in above there is $u_{13} \in \mathcal{C}^{1}\left([0, \mathrm{~m}], \mathcal{C}^{2}([2,3])\right)$ which is a solution to the problem (4.6) and satisfies the integral equation

$$
\begin{aligned}
& \int_{2}^{x} \int_{2}^{y}\left(u_{13}(t, z)-\phi(z)\right) d z d y \\
& \quad-\int_{0}^{t}\left(u_{13}(\tau, x)-u_{12}(\tau, 2)-(x-2) u_{12 x}(\tau, 2)\right) d \tau \\
& \quad-\int_{0}^{t} \int_{2}^{x} \int_{2}^{y} f\left(\tau, z, u_{13}(\tau, z), u_{13 x}(\tau, z)\right) d z d y d \tau \\
& =0, \quad t \in[0, m], \quad x \in[2,3] .
\end{aligned}
$$

The function

$$
u(t, x)=\left\{\begin{array}{lll}
u_{11}(t, x) & \text { in } & {[0, m] \times[0,1]} \\
u_{12}(t, x) & \text { in } & {[0, m] \times[1,2]} \\
u_{13}(t, x) & \text { in } & {[0, m] \times[2,3]}
\end{array}\right.
$$

is a $\mathcal{C}^{1}\left([0, \mathrm{~m}], \mathcal{C}^{2}([0,3])\right)$-solution to the problem

$$
\begin{gathered}
u_{t}-u_{x x}=f\left(t, x, u(t, x), u_{x}(t, x)\right) \text { in }[0, m] \times[0,3] \\
u(0, x)=\phi(x) \quad \text { in }[0,3] .
\end{gathered}
$$

An so on. We construct a solution $u_{1} \in \mathcal{C}^{1}\left([0, m], \mathcal{C}^{2}(\mathbb{R})\right)$ which is a solution to the problem

$$
\begin{gathered}
u_{t}-u_{x x}=f\left(t, x, u(t, x), u_{x}(t, x)\right) \text { in }(0, m] \times \mathbb{R}, \\
u(0, x)=\phi(x) \text { in } \mathbb{R} .
\end{gathered}
$$




\section{Proof of Theorem 1.4}

We have that the solution $u(t, x, \phi)$ satisfies the following integral equation

$$
\begin{aligned}
\mathrm{Q}(\phi)= & \int_{c}^{x} \int_{c}^{y}(u(t, z, \phi(z))-\phi(z)) d z d y \\
& -\int_{0}^{t}\left(u(\tau, x, \phi(x))-u(\tau, c, \phi(c))-(x-c) u_{x}(\tau, c, \phi(c))\right) d \tau \\
& -\int_{0}^{t} \int_{c}^{x} \int_{c}^{y} f\left(\tau, z, u(\tau, z, \phi(z)), u_{x}(\tau, z, \phi(z))\right) d z \\
= & 0, \quad t \in[0, m], \quad x \in[c, d] .
\end{aligned}
$$

Then

$$
\begin{aligned}
& \mathrm{Q}(\phi)-\mathrm{Q}\left(\phi_{1}\right)=\int_{\mathcal{c}}^{\mathrm{x}} \int_{\mathfrak{c}}^{y}\left(\mathrm{u}(\mathrm{t}, z, \phi(z))-\mathfrak{u}\left(\mathrm{t}, z, \phi_{1}(z)\right)-\left(\phi(z)-\phi_{1}(z)\right)\right) \mathrm{d} z \mathrm{~d} y \\
& -\int_{0}^{t}\left(u(\tau, x, \phi(x))-u\left(\tau, x, \phi_{1}(x)\right)\right) d \tau \\
& +\int_{0}^{t}\left(u(\tau, c, \phi(c))-u\left(\tau, c, \phi_{1}(c)\right)\right) d \tau \\
& +\int_{0}^{t}(x-c)\left(u_{x}(\tau, c, \phi(c))-u_{x}\left(\tau, c, \phi_{1}(c)\right)\right) d \tau \\
& -\int_{0}^{t} \int_{c}^{x} \int_{c}^{y}\left(f\left(\tau, z, u(\tau, z, \phi(z)), u_{x}(\tau, z, \phi(z))\right)\right. \\
& \left.-\mathrm{f}\left(\tau, z, u\left(\tau, z, \phi_{1}(z)\right), u_{x}\left(\tau, z, \phi_{1}(z)\right)\right)\right) \mathrm{d} z \mathrm{~d} y \mathrm{~d} \tau \\
& =\int_{\mathfrak{c}}^{x} \int_{c}^{y}\left(\frac{\partial u}{\partial \phi}(t, z, \phi(z))-1\right) d z d y \\
& -\int_{0}^{t} \frac{\partial u}{\partial \phi}(\tau, x, \phi(x)) d \tau+\int_{0}^{t} \frac{\partial u}{\partial \phi}(\tau, c, \phi(c)) d \tau+\int_{0}^{t}(x-c)\left(\frac{\partial u}{\partial \phi}\right)_{x}(\tau, c, \phi(c)) d \tau \\
& -\int_{0}^{t} \int_{\mathcal{c}}^{x} \int_{\mathcal{c}}^{y} \frac{\partial f}{\partial u}\left(\tau, z, u(\tau, z, \phi(z)), u_{x}(\tau, z, \phi(z))\right) \frac{\partial u}{\partial \phi}(\tau, z, \phi(z)) d z d y d \tau \\
& -\int_{0}^{t} \int_{c}^{x} \int_{c}^{y} \frac{\partial f}{\partial u_{x}}\left(\tau, z, u(\tau, z, \phi(z)), u_{x}(\tau, z, \phi(z))\right)\left(\frac{\partial u}{\partial \phi}\right)_{x}(\tau, z, \phi(z)) d z d y d \tau \\
& +\delta\left\{\phi, \phi_{1}\right\},
\end{aligned}
$$


where $\delta\left\{\phi, \phi_{1}\right\} \longrightarrow 0$ as $\phi(x) \longrightarrow \phi_{1}(x)$ for every $x \in[c, d]$. Hence, when $\phi(x) \longrightarrow \phi_{1}(x)$ for every $x \in[c, d]$, we get

$$
\begin{aligned}
0= & \int_{c}^{x} \int_{c}^{y}(v(t, z)-1) d z d y-\int_{0}^{t} v(\tau, x) d \tau \\
& +\int_{0}^{t} v(\tau, c) d \tau+\int_{0}^{t} x v_{x}(\tau, c) d \tau \\
& -\int_{0}^{t} \int_{c}^{x} \int_{c}^{y} \frac{\partial f}{\partial u}\left(\tau, z, u(\tau, z, \phi(z)), u_{x}(\tau, z, \phi(z))\right) v(\tau, z) d z d y d \tau \\
& -\int_{0}^{t} \int_{c}^{x} \int_{c}^{y} \frac{\partial f}{\partial u_{x}}\left(\tau, z, u(\tau, z, \phi(z)), u_{x}(\tau, z, \phi(z))\right) v_{x}(\tau, z) d z d y d \tau,
\end{aligned}
$$

which we differentiate twice in $x$ and once in $t$ and we get that $v$ satisfies (1.3). Now we put $t=0$ in (5.1) and then we differentiate twice in $x$, and we find that $v$ satisfies (1.4).

\section{Acknowledgments}

The authors would like to thank the anonymous referees for their helpful comments and suggestions.

\section{References}

[1] A. Braik, A. Beniani and Kh. Zennir Polynomial stability for system of 3 wave equations with infinite memories, Math. Meth. Appl. Sci. 2017; 115. DOI: 10.1002/mma.4599

[2] A. Braik, Y. Miloudi and Kh. Zennir A finite-time blow-up result for a class of solutions with positive initial energy for coupled system of heat equations with memories, Math. Meth. Appl. Sci. 2017; 19. DOI: 10.1002/mma.4695

[3] K.Deng, Comparison principle for some nonlocal problems, Quart. Appl. Math. 50 (1992), no. 3,517522 .

[4] Z.B.Fang and J. Zhang, Global and blow-up solutions for the nonlocal p-Laplacian evolution equationwith weighted nonlinear nonlocal boundary condition, J. Integral Equat. Appl. 26 (2014), no. 2, 171196.

[5] Y.Gao and W.Gao, Existence and blow-up of solutions for a porous medium equation with nonlocal boundary condition, Appl. Anal. 90 (2011), no. 5, 799809.

[6] A.Gladkov and M.Guedda, Blow-up problem for semilinear heat equation with absorption and a nonlocal boundary condition, Nonlinear Anal. 74 (2011), no. 13, 45734580.

[7] A.Gladkov and M.Guedda, Semilinear heat equation with absorption and a nonlocal boundary condition, Appl. Anal. 91 (2012), no. 12, 22672276.

[8] A.Gladkov and K. I.Kim, Blow-up of solutions for semilinear heat equation with nonlinear nonlocal boundary condition, J. Math. Anal. Appl. 338 (2008), 264273. 
[9] A.Gladkov and K. I.Kim, Uniqueness and nonuniqueness for reaction-diffusion equation with nonlocal boundary condition, Adv. Math. Sci. Appl. 19 (2009), no. 1, 3949.

[10] A.Gladkov and A.Nikitin, A reaction-diffusion system with nonlinear nonlocal boundary conditions, Int. J. Partial Differential Equations 2014 (2014), Article ID 523-656.

[11] D. Liu, Blow-up for a degenerate and singular parabolic equation with nonlocal boundary condition, J. Nonlinear Sci. Appl. 9 (2016), 208218.

[12] D. Liu and C.Mu, Blowup properties for a semilinear reaction-diffusion system with nonlinear nonlocal boundary conditions, Abstr. Appl. Anal. 2010 (2010), Article ID 148035, 1-17.

[13] A. Samarskii, V. Galaktionov, S. Kurdyumov and A. Mikhailov. Blow-up in Quasilinear Parabolic Equations. Walter de Gruyer-Berlin-New York. 1995.

[14] T. Xiang and R. Yuan. A class of expansive type Krasnoselskii fixed point theorem. Nonlinear Analysis, 2009, pp. 3229-3239.

[15] G. Zhong and L.Tian Blow up problems for a degenerate parabolic equation with nonlocal source and nonlocal nonlinear boundary condition, Boundary Value Problems 2012 (2012), no. $45,1-14$.

[16] J. Zhou and D.Yang Blowup for a degenerate and singular parabolic equation with nonlocal source and nonlocal boundary, Appl. Math. Comput. 256 (2015), 881-884. 